\title{
Rumor-Propagation Model with Consideration of Refutation Mechanism in Homogeneous Social Networks
}

\author{
Laijun Zhao, ${ }^{1,2}$ Xiaoli Wang, ${ }^{3}$ Jiajia Wang, ${ }^{1,2}$ Xiaoyan Qiu, ${ }^{4}$ and Wanlin Xie ${ }^{4}$ \\ ${ }^{1}$ Sino-US Global Logistics Institute, Shanghai Jiao Tong University, Shanghai 200030, China \\ ${ }^{2}$ Antai College of Economics \& Management, Shanghai Jiao Tong University, Shanghai 200052, China \\ ${ }^{3}$ School of Management, Shanghai University of Engineering Science, Shanghai 201620, China \\ ${ }^{4}$ School of Management, Shanghai University, Shanghai 200444, China \\ Correspondence should be addressed to Xiaoli Wang; wxl-0539@163.com
}

Received 26 March 2014; Revised 19 July 2014; Accepted 19 July 2014; Published 18 August 2014

Academic Editor: Cengiz Çinar

Copyright (C) 2014 Laijun Zhao et al. This is an open access article distributed under the Creative Commons Attribution License, which permits unrestricted use, distribution, and reproduction in any medium, provided the original work is properly cited.

\begin{abstract}
In recent years, increasing attention has been paid to how to effectively manage rumor propagation. Based on previous studies of rumor propagation and some strategies used by the authorities to refute rumors and manage rumor propagation, we develop a new rumor-propagation model with consideration of refutation mechanism. In this paper, we describe the dynamic process of rumor propagation by accounting for the refutation mechanism in homogeneous social networks. And then, we derive mean-field equations for rumor-propagation process. We then analyze the stability of the model with respect to changes in parameter values. Our results show that there exists a critical threshold $\lambda_{c}$ that is inversely proportional to the average degree of the social networks and is positively correlated with the strength of the refutation mechanism. If the spreading rate is bigger than the critical threshold $\lambda_{c}$, rumors can be spread. Our numerical simulations in homogeneous networks demonstrate that increasing the ignorant's refutation rate $\beta$ can reduce the peak value of spreaders density, which is better than increasing the spreader's refutation rate $\eta$. Therefore, based on the seriousness of the rumor propagation and the rumor-propagation rate, the authorities can choose effective strategies that increase the refutation rate so that they can reduce the maximum influence of the rumor
\end{abstract}

\section{Introduction}

The propagation of rumors is a typical form of social interaction that exerts a powerful influence on human affairs [13]. Although "rumors" can also imply truthful information, most rumors contain some degree of falsehood, and it is these false rumors that we will focus on in this paper. With the development of technologies such as the Internet and the emergence of new media (especially blogging and Twitter), rumors can spread faster and farther than ever before. When an emergency occurs, the subsequent rumor propagation may lead to more serious harm than the emergency itself [46]. For instance, in March 2011, a buying frenzy for iodized salt erupted in China because of the rumor that iodized salt could protect people from the nuclear radiation released as a result of damage to Japan's Fukushima nuclear plant caused by the Tohoku earthquake. This behavior not only damaged the normal market order but also negatively affected the daily life of many citizens.

The study of rumor-propagation models began in the 1960s. The first classical rumor model (the DK model) was proposed by Daley and Kendall [7]. Afterwards, Maki and Thompson modified the DK model and developed another model that also became a classic in this field [8]. In both models, people are divided into three groups: people who know and transmit a rumor, people who do not know the rumor yet, and people who know the rumor but choose not to transmit it to others. Several variants of these models were subsequently developed by other researchers [9-12].

Sudbury [11] studied the dynamic mechanisms of information transmission in social networks and proposed that the behavior of rumor propagation could be described by what they called the "SIR" model. In this model, a closed and homogeneous population is divided into three groups: 
ignorants (I, who have not yet heard of the rumor), spreaders ( $S$, who spread the rumor), and stiflers ( $R$, who have heard the rumor but have ceased to spread it). Some rules for the rumor-propagation process can be described based on these categories. When a spreader contacts an ignorant, the ignorant could become a spreader at a certain rate. When a spreader contacts another spreader or a stifler, the former spreader could turn into a stifler at a certain rate. The rumor dies out when no spreaders remain in the population.

Many other scholars have studied rumor propagation by considering the topological properties of social networks [13-16]. Zanette [17, 18] and Buzna et al. [19] studied the dynamic process of rumor propagation and found that there was a critical rumor-propagation threshold that must be exceeded before a rumor can propagate in small-world networks. Moreno et al. [20] examined the dynamics of the critical rumor-propagation model for complex networks. Gu and $\mathrm{Cai}$ [21] and $\mathrm{Gu}$ et al. [22] introduced forgetting and remembering functions in a two-state model. Nekovee et al. [23] and Zhao et al. [24] studied rumor-propagation models that included a forgetting mechanism. Zhao et al. [25, 26] refined these models considering both forgetting and remembering mechanisms in complex networks. Zhao et al. [27] studied rumor-propagation model in BBV networks and derived the corresponding mean-field based on the strength of the nodes. To characterize information propagation on online microblogs, Xiong et al. [28] proposed a diffusion model (SCIR), which contains four possible states: susceptible, contacted, infected, and refractory. Cheng et al. [29] introduced a stochastic epidemic model of the rumor diffusion, in which the infectious probability is defined as a function of the strength of ties.

The spread of rumors has always had the potential to lead to serious harm, but this potential has increased due to the ability of rumors to spread rapidly through modern social networks. The potential harm caused by rumor propagation has therefore attracted the attention of many countries. To minimize this harm, the authorities have adopted various measures to manage the spread of rumors. For example, in some countries, such as the United States, England, and China, special websites exist to refute rumors; these include http://www.sunnidefense.com/exp/, http://snopes.com/, and http://baike.m4.cn/. When rumor propagation occurs, the government should take immediate measures to refute the rumor and provide a smooth flow of correct information to the people, thereby helping them to learn the truth. This approach can greatly reduce the influence of rumors. This means that rumor-propagation models should account for the strategies adopted by the authorities to refute rumors. As far as the refutation mechanism is concerned, an ignorant who is contacted by a rumor spreader will become a stifler at a certain rate, because the ignorant's ability to distinguish true information from the false is improved by the strategies adopted by the authorities to refute rumors; at the same time, under the refutation mechanism, rumor spreaders may spontaneously become stiflers at a certain rate because the spreaders become aware of the truth of the rumor. However, no previous models consider the refutation mechanism.

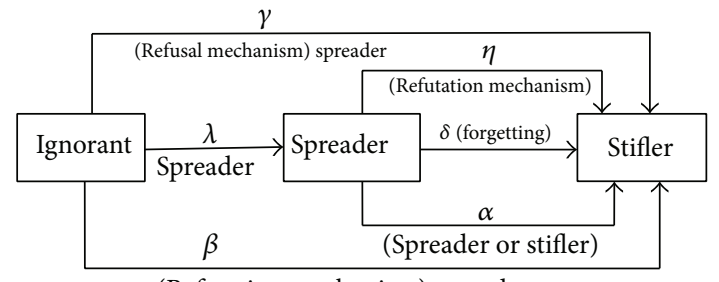

(Refutation mechanism) spreader

FIgURE 1: Structure of the rumor-propagation process in a model that incorporates a refutation mechanism.

A model that considers the refutation mechanism would clearly describe rumor propagation more accurately than a model that lacks such a mechanism. Thus, in the present study, we examine the dynamic process of rumor propagation by considering the impact of the refutation mechanism. The paper is organized as follows. In Section 2, we develop a rumor-propagation model that incorporates a refutation mechanism and provide the corresponding mean-field equations. We present analytical results for a steady state in this model in Section 3 and in Section 4 we describe numerical simulations of the dynamics of the model to analyze the impact of various factors on the model's outputs. In Section 5, we conclude this paper by summarizing the key results of our analysis and providing an outlook for future research.

\section{An SIR Rumor-Propagation Model That Incorporates a Refutation Mechanism}

The propagation of rumors is a complex sociopsychological process. In this paper, we define our model based on certain simplifying initial assumptions. We assume that a closed social network exists, in which there is uniform mixing of the $N$ individuals in the network. These individuals can be represented as vertices in the network, and links between them can be represented as edges. This model provides an undirected graph $G=(\mathbf{V}, \mathbf{E})$, in which $\mathbf{V}$ is the set of vertices and $\mathbf{E}$ is the set of edges. Based on the work of Maki and Thompson [8], we assume that a rumor propagates through direct contacts between the spreader and others in the social network. Figure 1 illustrates the propagation process in a network in which the population is divided into ignorants, spreaders, and stiflers. The rumor-propagation rules can be summarized as follows.

(1) When an ignorant contacts a rumor spreader, the ignorant becomes a rumor spreader at rate $\lambda$, which we define as the rumor-propagation rate.

(2) Based on the internal discrimination ability of the ignorants from their own hobbies, knowledge backgrounds, and experiences, we assume that when an ignorant contacts a spreader, the ignorant becomes a stifler at rate $\gamma$, which we define as the refusal rate, where we do not consider the refutation strategies used by the authorities; that is, the ignorant's ability to distinguish true information from the false is not 
affected by the external strategies adopted by the authorities to refute rumors.

(3) To account for the refutation strategies issued by the authorities, we assume that an ignorant who is contacted by a rumor spreader will become a stifler at a certain rate $\beta$, because the ignorant's ability to distinguish true information from the false is improved by the authorities' refutation strategies; thus, we define the parameter $\beta$ as ignorant's refutation rate.

(4) Under the refutation mechanism, rumor spreaders may also cease spreading a rumor at a certain rate $\eta$, because the spreaders become aware of the truth of the rumor. Thus, we define the parameter $\eta$ as spreader's refutation rate.

(5) When a rumor spreader contacts another rumor spreader or a stifler, the former rumor spreader may become a stifler at a rate $\alpha$, which we define as the stifling rate.

(6) Based on differences in the personality, knowledge background, and experience of the spreaders, we account for the forgetting mechanism by assuming that rumor spreaders may also cease spreading a rumor spontaneously at rate $\delta$, which we define as the forgetting rate.

In the paper, $\gamma$ and $\beta$ represent different influences of internal factor and external factor, respectively.

Because the parameters $\lambda, \beta, \eta, \gamma, \alpha$, and $\delta$ are rates, their values cannot be smaller than zero.

Many social networks are abstracted as homogenous networks, in which degree fluctuations are very small and there are no degree correlations. The degree of nodes on homogeneous networks has a Poisson distribution; that is to say, the above networks degrees distributions peak at an average value and show small fluctuation around the value. That is, in homogeneous networks, most nodes have the same degree, whose value is approximately equal to the average degree of network. The rumor propagation follows the above rules in homogeneous networks and each node has the same degree $\langle k\rangle$. In the model that incorporates a refutation mechanism, $\mathrm{I}(t), \mathrm{S}(t)$, and $\mathrm{R}(t)$ denote the proportions of the population that are ignorants, spreaders, and stiflers, respectively, at time $t$. These proportions satisfy the normalization condition: $\mathrm{I}(t)+\mathrm{S}(t)+\mathrm{R}(t)=1$. According to the above elaborated rumor spreading progress, the meanfield equations for our model in homogeneous networks can be described as follows:

$$
\begin{aligned}
\frac{d \mathrm{I}(t)}{d t}= & -\lambda\langle k\rangle \mathrm{I}(t) \mathrm{S}(t)-\beta\langle k\rangle \mathrm{S}(t) \mathrm{I}(t) \\
& -\gamma\langle k\rangle \mathrm{S}(t) \mathrm{I}(t), \\
\frac{d \mathrm{~S}(t)}{d t}= & \lambda\langle k\rangle \mathrm{I}(t) \mathrm{S}(t)-\alpha\langle k\rangle \mathrm{S}(t)[\mathrm{S}(t)+\mathrm{R}(t)] \\
& -\eta \mathrm{S}(t)-\delta \mathrm{S}(t),
\end{aligned}
$$

$$
\begin{aligned}
\frac{d \mathrm{R}(t)}{d t}= & \alpha\langle k\rangle \mathrm{S}(t)[\mathrm{S}(t)+\mathrm{R}(t)]+\delta \mathrm{S}(t) \\
& +\beta\langle k\rangle \mathrm{S}(t) \mathrm{I}(t)+\eta \mathrm{S}(t)+\gamma\langle k\rangle \mathrm{S}(t) \mathrm{I}(t) .
\end{aligned}
$$

Equation (1) indicates that the density of ignorants varies over time $t$, which consists of three parts: the density of ignorants varies over time $t$ caused by ignorants contacting spreaders and ignorants becoming spreaders at the rate $\lambda$; the density of ignorants varies over time $t$ caused by ignorants contacting spreaders and ignorants becoming stiflers at the rate $\gamma$ due to refusal mechanism; the density of ignorants varies over time $t$ caused by ignorants contacting spreaders and ignorants becoming stiflers at the rate $\beta$ due to refutation mechanism. Equation (2) indicates that the density of spreaders varies over time $t$, which consists of four parts: the density of spreaders varies over time $t$ caused by ignorants contacting spreaders and ignorants becoming spreaders at the rate $\lambda$; the density of spreaders varies over time $t$ caused by spreaders contacting other spreaders or stiflers, or the former spreaders may become stiflers at the rate $\alpha$; the density of spreaders varies over time $t$ caused by spreaders that may also become stiflers at the rate $\eta$ due to refutation mechanism; the density of spreaders varies over time $t$ caused by spreaders that may also become stiflers at the rate $\delta$ due to forgetting mechanism. Equation (3) indicates that the density of stiflers varies over time $t$, which consists of five parts: the density of stiflers varies over time $t$ caused by spreaders contacting other spreaders or stiflers, and the former spreaders may become stiflers at the rate $\alpha$; the density of stiflers varies over time $t$ caused by spreaders that may also become stiflers at the rate $\delta$ due to forgetting mechanism; the density of stiflers varies over time $t$ caused by ignorants contacting spreaders and ignorants becoming stiflers at the rate $\beta$ due to refutation mechanism; the density of stiflers varies over time $t$ caused by spreaders that may also become stiflers at the rate $\eta$ due to refutation mechanism; the density of stiflers varies over time $t$ caused by ignorants contacting spreaders and ignorants becoming stiflers at the rate $\gamma$ due to refusal mechanism.

The classical SIR rumor-spread model [7] and the classical SIR rumor-spread model that incorporates a forgetting mechanism [23] are special cases of our new version of the SIR model that includes a refutation mechanism.

Case 1. When $\beta=0, \delta=0, \eta=0$, and $\gamma=0$, the model becomes the classical SIR rumor-spread model [7].

Case 2. When $\beta=0, \delta \neq 0, \eta=0$, and $\gamma=0$, the model becomes Nekovee's rumor-spread model, which incorporates a forgetting mechanism [23].

\section{Steady-State Analysis}

During the process of rumor propagation in a homogeneous network, we assume that there is initially one spreader and that the rest of the people are ignorants. The initial condition for rumor-propagation at time $t=0$ is $\mathrm{I}(0)=(N-1) / N$, $\mathrm{S}(0)=1 / N$, and $\mathrm{R}(0)=0$.

The number of spreaders first increases and then decreases until it reaches zero, at which point the rumor stops 
spreading. Finally, when the system reaches a stable state, there are no spreaders and only ignorants and stiflers remain. We analyze the final size of a rumor $\mathrm{R}$, which can be utilized to measure the level of rumor influence. Here the final rumor size $\mathrm{R}=\lim _{t \rightarrow \infty} \mathrm{R}(t)=\mathrm{R}(\infty)$. We will discuss this in more detail in this section.

We calculate the final rumor size, $\mathrm{R}$, by analyzing the mean-field equations in homogeneous networks. Dividing (1) by (3) will get

$$
\begin{aligned}
\frac{d \mathrm{R}(t)}{d \mathrm{I}(t)}= & (\alpha\langle k\rangle \mathrm{S}(t)[\mathrm{S}(t)+\mathrm{R}(t)]+\delta \mathrm{S}(t) \\
& +\beta\langle k\rangle \mathrm{S}(t) \mathrm{I}(t) \\
& +\eta \mathrm{S}(t)+\gamma\langle k\rangle \mathrm{S}(t) \mathrm{I}(t)) \\
& \times(-\lambda\langle k\rangle \mathrm{I}(t) \mathrm{S}(t)-\beta\langle k\rangle \mathrm{S}(t) \mathrm{I}(t) \\
= & \frac{(\alpha\langle k\rangle+\delta+\eta) \mathrm{S}(t)}{(-\lambda-\beta-\gamma)\langle k\rangle \mathrm{S}(t) \mathrm{I}(t)} \\
& +\frac{(\beta+\gamma-\alpha)\langle k\rangle \mathrm{S}(t) \mathrm{I}(t)}{(-\lambda-\beta-\gamma)\langle k\rangle \mathrm{S}(t) \mathrm{I}(t)} \\
= & \frac{(\alpha\langle k\rangle+\delta+\eta)}{(-\lambda-\beta-\gamma)\langle k\rangle \mathrm{I}(t)}+\frac{\beta+\gamma-\alpha}{-\lambda-\beta-\gamma} .
\end{aligned}
$$

Then,

$$
d \mathrm{R}(t)=\frac{(\alpha\langle k\rangle+\delta+\eta) d \mathrm{I}(t)}{(-\lambda-\beta-\gamma)\langle k\rangle \mathrm{I}(t)}+\frac{\beta+\gamma-\alpha}{-\lambda-\beta-\gamma} d \mathrm{I}(t)
$$

Both sides of (5) can be integrated with respect to $\mathrm{R}(t)$ and $\mathrm{I}(t)$ from the initial state to the stable state. Considering the initial condition $\mathrm{I}(0)=(N-1) / N(N \rightarrow \infty)$ and the final state for the ignorants $\lim _{t \rightarrow \infty} \mathrm{I}(t)=1-\lim _{t \rightarrow \infty} \mathrm{R}(t)=1-\mathrm{R}$. We can therefore get

$$
\begin{aligned}
\left.\mathrm{R}(t)\right|_{0} ^{\infty}= & \left.\frac{\alpha\langle k\rangle+\delta+\eta}{(-\lambda-\beta-\gamma)\langle k\rangle} \ln \mathrm{I}(t)\right|_{0} ^{\infty} \\
& +\left.\frac{\beta+\gamma-\alpha}{-\lambda-\beta-\gamma} \mathrm{I}(t)\right|_{0} ^{\infty}, \\
\mathrm{R}= & \frac{\alpha\langle k\rangle+\delta+\eta}{(-\lambda-\beta-\gamma)\langle k\rangle}[\ln (1-\mathrm{R})] \\
& +\frac{\beta+\gamma-\alpha}{\lambda+\beta+\gamma} \mathrm{R} .
\end{aligned}
$$

Taking the logarithm of $e$ for (7), we obtain the following transcendental equation:

$$
\mathrm{R}=1-e^{-\varepsilon \mathrm{R}}
$$

where

$$
\varepsilon=\frac{(\lambda+\alpha)\langle k\rangle}{(\delta+\alpha\langle k\rangle+\eta)}>1 .
$$

Given

$$
f(\mathrm{R})=1-e^{-\varepsilon \mathrm{R}}-\mathrm{R}=1-e^{(-(\lambda+\alpha)\langle k\rangle /(\delta+\alpha\langle k\rangle+\eta)) \mathrm{R}}-\mathrm{R},
$$

taking the derivation of $f(\mathrm{R})$ with respect to $\mathrm{R}$, we have

$$
\begin{gathered}
f^{\prime}(\mathrm{R})=\frac{(\lambda+\alpha)\langle k\rangle}{(\delta+\alpha\langle k\rangle+\eta)} e^{(-(\lambda+\alpha)\langle k\rangle /(\delta+\alpha\langle k\rangle+\eta)) \mathrm{R}}-1 \\
f^{\prime \prime}(\mathrm{R})=-\left[\frac{(\lambda+\alpha)\langle k\rangle}{(\delta+\alpha\langle k\rangle+\eta)}\right]^{2} e^{(-(\lambda+\alpha)\langle k\rangle /(\delta+\alpha\langle k\rangle+\eta)) \mathrm{R}} \\
-\left[\frac{(\lambda+\alpha)\langle k\rangle}{(\delta+\alpha\langle k\rangle+\eta)}\right]^{2}<0, \quad e^{(-(\lambda+\alpha)\langle k\rangle /(\delta+\alpha\langle k\rangle+\eta)) \mathrm{R}}>0 \\
f^{\prime \prime}(\mathrm{R})<0 .
\end{gathered}
$$

That is to say, $f(\mathrm{R})$ is a concave function on the interval $[0,1)$. Also $f(0)=0$ and

$$
\begin{gathered}
f^{\prime}(0)=\frac{(\lambda+\alpha)\langle k\rangle}{(\delta+\alpha\langle k\rangle+\eta)}-1=\frac{\lambda\langle k\rangle-\eta-\delta}{(\delta+\alpha\langle k\rangle+\eta)}, \\
\lim _{\mathrm{R} \rightarrow 1^{-}} f(\mathrm{R})=\lim _{\mathrm{R} \rightarrow 1^{-}}\left[1-e^{(-(\lambda+\alpha)\langle k\rangle /(\delta+\alpha\langle k\rangle+\eta)) \mathrm{R}}-\mathrm{R}\right] \\
=-e^{-(\lambda+\alpha)\langle k\rangle /(\delta+\alpha\langle k\rangle+\eta)}<0 .
\end{gathered}
$$

So, $f(\mathrm{R})$ has a nontrivial solution and the following condition must be fulfilled: $f^{\prime}(0)>0$; that is,

$$
\left.\frac{d}{d \mathrm{R}}\left(1-e^{-\varepsilon \mathrm{R}}\right)\right|_{\mathrm{R}=0}>1,
$$

where

$$
\varepsilon=\frac{(\lambda+\alpha)\langle k\rangle}{(\delta+\alpha\langle k\rangle+\eta)}>1 .
$$

That is, (8) permits a nonzero solution only if $\varepsilon>1$. For $\delta \neq 0$, this condition is fulfilled if the following condition is true:

$$
\frac{\lambda\langle k\rangle}{\delta+\eta}>1
$$

This condition is equivalent to the constraint $\lambda>$ $\lambda_{c}=(\delta+\eta) /\langle k\rangle$, which represents the critical rumorpropagation threshold that must be exceeded before a rumor can propagate. In particular, for $\delta=1$, we obtain the critical rumor-propagation threshold in a homogeneous network:

$$
\lambda_{c}=\frac{1+\eta}{\langle k\rangle} .
$$

This result shows that the threshold for rumor propagation is not only related to the average degree $\langle k\rangle$ of the social network but also depends on the $\eta$ refutation rate. From (19), we know that, given a fixed $\langle k\rangle$, the critical rumorpropagation threshold $\lambda_{c}$ increases with increasing $\eta$. That is, rumor propagation becomes more difficult with a strong refutation mechanism. 


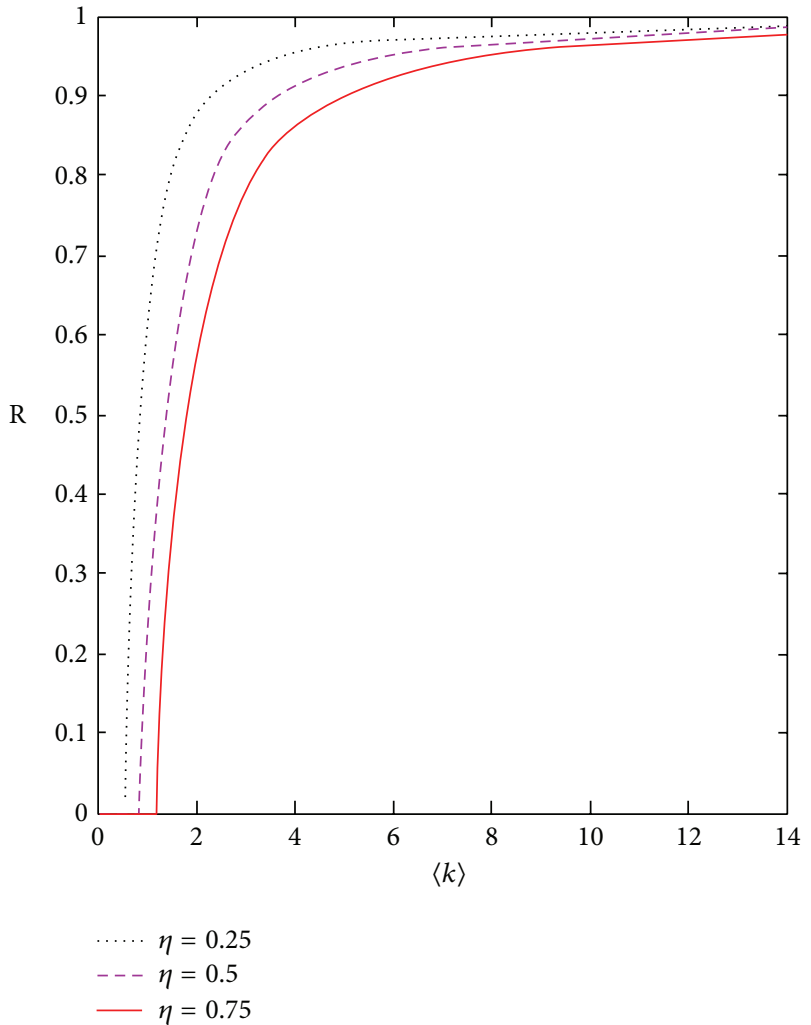

(a)

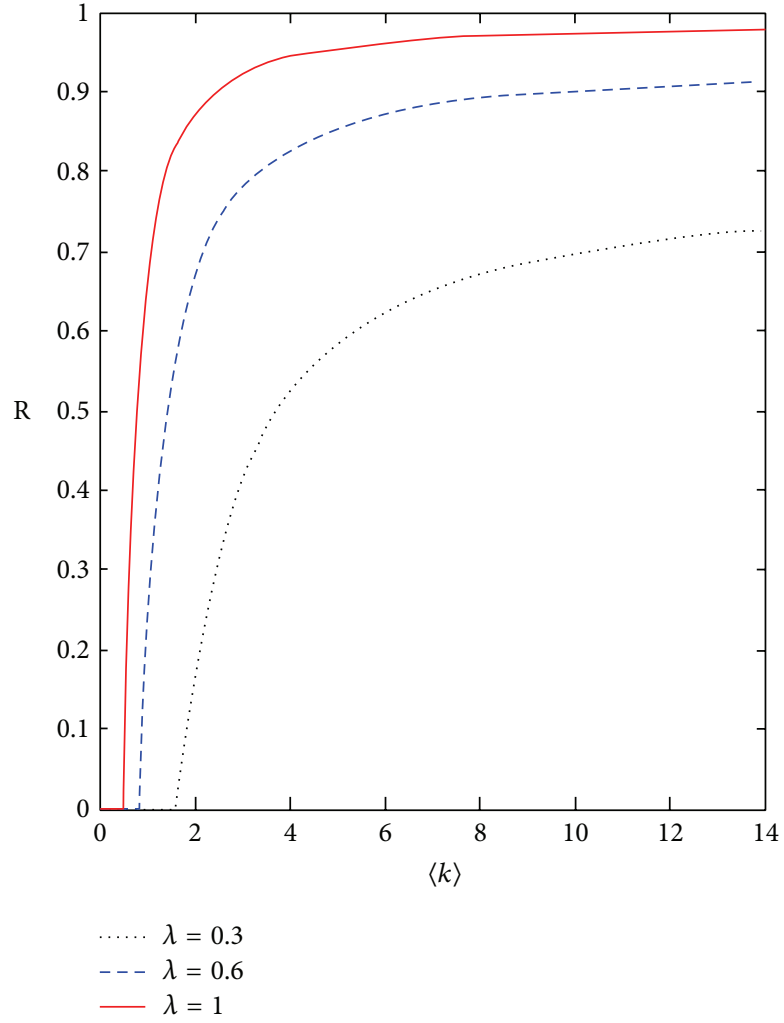

(b)

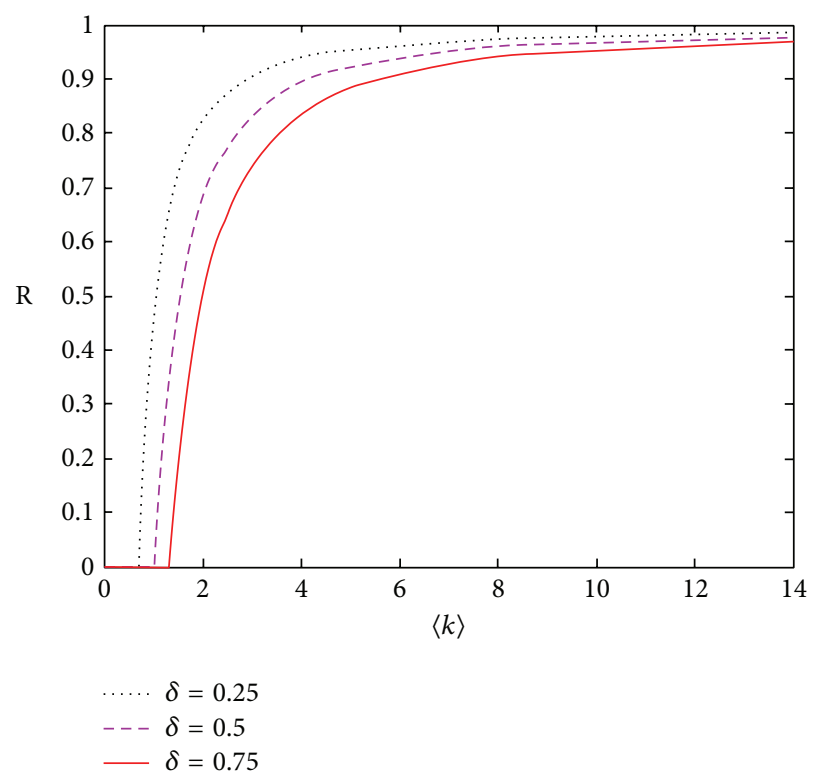

(c)

FIGURE 2: The relationship between rumor influence $\mathrm{R}$ and $\langle k\rangle$ for various combinations of rates. (a) The effect of the $\eta$ on $\mathrm{R}$ for $\lambda=0.8$, $\alpha=0.2$, and $\delta=0.2$. (b) The effect of $\lambda$ on $\mathrm{R}$ for $\delta=0.3, \alpha=0.3$, and $\eta=0.2$. (c) The relationship between $\delta$ and $\mathrm{R}$ for $\lambda=0.8, \alpha=0.2$, and $\eta=0.3$.

\section{Numerical Solution}

First, according to (7), we analyze the effects of various factors on $\mathrm{R}$ by using Matlab. Figure 2 displays the variation of $\mathrm{R}$ as a function of $\langle k\rangle$ with refutation rate $\eta$, propagation rate $\lambda$, and forgetting rate $\delta$, respectively. Figure 2(a) shows how $\mathrm{R}$ changes for three values of $\eta$. As the value of $\eta$ increases, $\mathrm{R}$ decreases; as the value of $\langle k\rangle$ increases, $\mathrm{R}$ increases and 


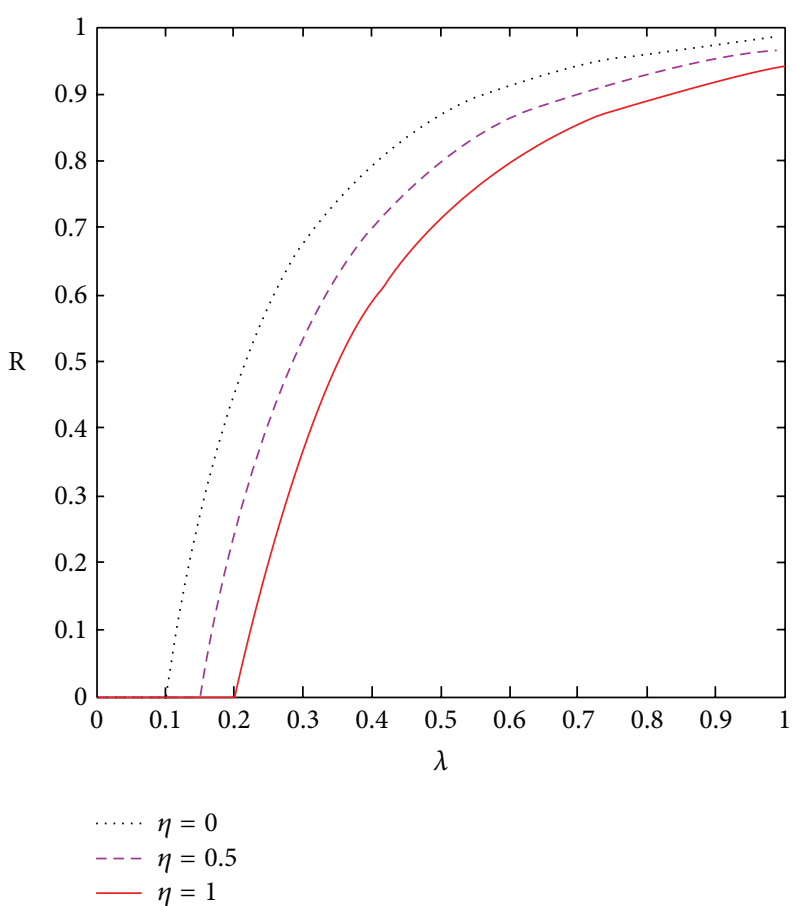

(a)

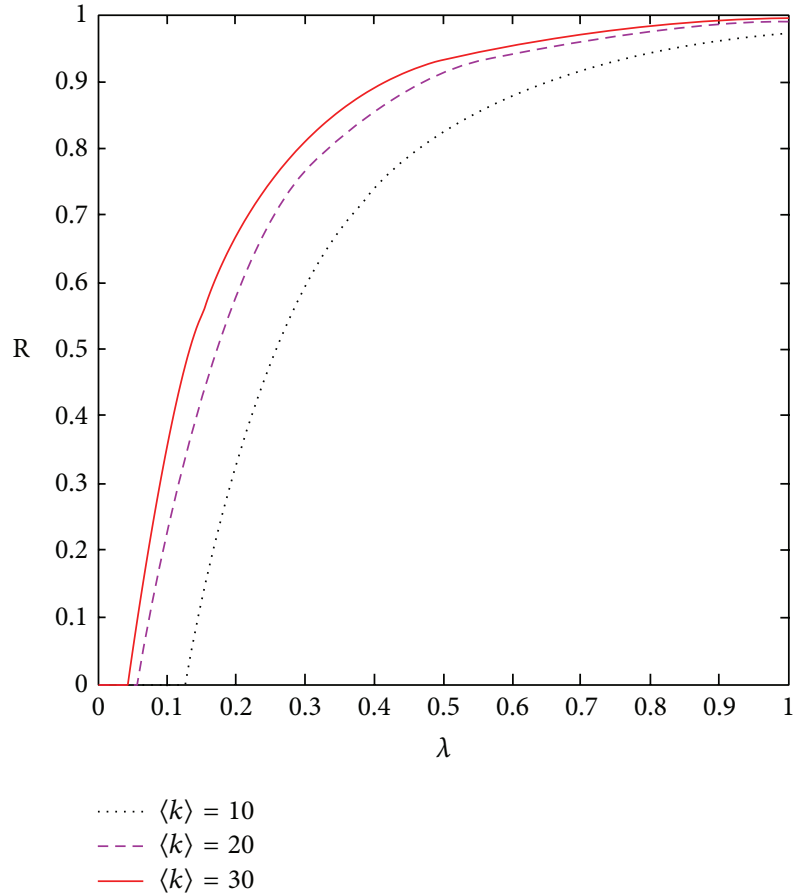

(b)

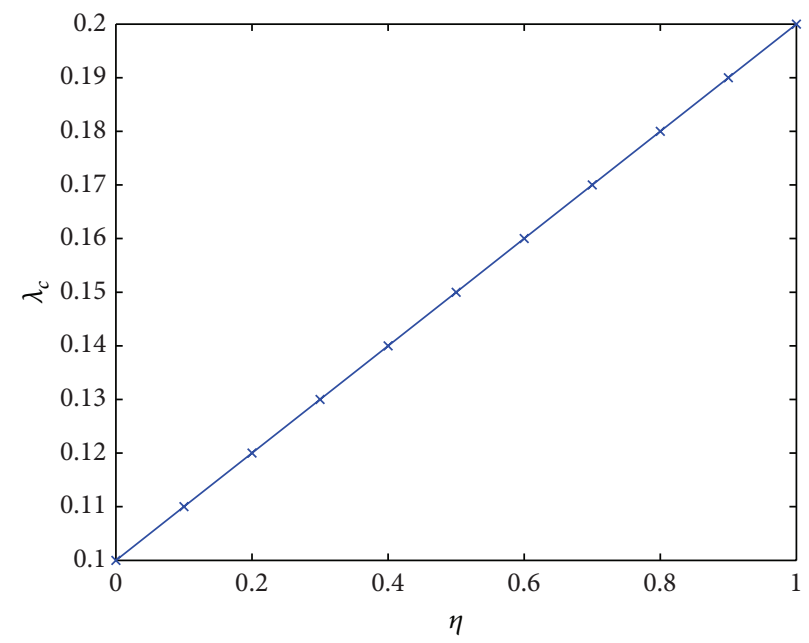

(c)

FIGURE 3: The relationship between the final size of $\mathrm{R}$ as a function of $\lambda$ with $\delta=1$ and the relationship between the rumor spreading threshold $\lambda_{c}$ and $\eta$. (a) The effect of $\eta$ on the relationship for $\langle k\rangle=10$ and $\alpha=0.2$. (b) The effect of $\langle k\rangle$ on the relationship for $\alpha=0.2$ and $\eta=0.3$. (c) The relationship between the rumor spreading threshold $\lambda_{c}$ and $\eta$ with $\langle k\rangle=0.3, \alpha=0.2$.

eventually reaches a steady state that depends on the value of $\eta$. These results indicate that adding $\eta$ to the model affects the final size of the rumor, R. Figure 2(b) reveals that the larger the propagation rate, the greater the value of R. Figure 2(c) reveals that the forgetting rate $\delta$ has noticeable influence on rumor propagation. The larger the forgetting rate, the smaller the value of $\mathrm{R}$ at a given value of $\langle k\rangle$. For a given forgetting rate, $\mathrm{R}$ increases as $\langle k\rangle$ increases, eventually reaching a steady state.

At the steady state, we can obtain a critical rumor threshold $\lambda_{c}$. In particular, for $\delta=1$, we can obtain the critical rumor-propagation threshold $\lambda_{c}$, which depends on the average degree $\langle k\rangle$ of the social network and the $\eta$ refutation rate. Figure 3 confirms this conclusion. According to (7), we can plot Figures 3(a), 3(b), and 3(c) by using Matlab. Figure 3(a) shows how $\mathrm{R}$ changes as a function of the spreading rate $\lambda$ for three values of $\eta$, with $\langle k\rangle=10$ and $\alpha=$ 0.2 . According to (11), when $\langle k\rangle=10, \alpha=0.2$, and $\eta=0$, we can calculate that the critical rumor-propagation threshold is $\lambda_{c}=0.1$. Similarly we can obtain other thresholds of $\lambda_{c}=$ 0.15 and $\lambda_{c}=0.2$ at $\eta=0.5$ and $\eta=1$, respectively. The above conclusions are verified by simulation data in Figure 3(a). 
Figure 3(a) clearly shows that when the propagation rate is larger than the critical rumor-propagation threshold (i.e., when $\lambda>\lambda_{c}$ ), a rumor can spread. Figure 3(a) also shows that, as $\eta$ increases, $\mathrm{R}$ decreases. Figure $3(\mathrm{~b})$ reveals how $\mathrm{R}$ changes as a function of $\lambda$ for three values of $\langle k\rangle$, with $\alpha=0.2$ and $\eta=0.3$. Following the same approach, we can obtain the corresponding rumor-propagation thresholds. As $\langle k\rangle$ increases, $\lambda_{c}$ decreases. Figure 3(c) shows the relationship between $\lambda_{c}$ and $\eta$ under the given conditions. That is, $\lambda_{c}$ increases as $\eta$ increases. Analytical result (see (11)) is verified by the simulation in Figure 3.

At last, based on the descriptions in Section 2, we constructed a homogeneous network consisting of $N=10^{6}$ nodes, with an average degree $\langle k\rangle=10$, with each person representing a vertex in the network and each path between two people representing an edge. In the following simulations, we assumed an initial condition with only one spreader in the homogeneous network; thus $\mathrm{I}(0)=\left(10^{6}-1\right) / 10^{6}, \mathrm{~S}(0)=$ $1 / 10^{6}$, and $\mathrm{R}(0)=0$. The fourth-order Runge-Kutta method is widely used to solve the differential equation problem. So we can use the fourth-order Runge-Kutta method to solve the coupled differential equations (1) to (3), and we performed numerical simulations to analyze the effects of the model parameters on the rumor-propagation process. The concrete steps of the algorithm are given as follows:

$$
\begin{gathered}
\mathrm{I}_{n+1}(t)=\mathrm{I}_{n}(t)+\frac{h}{6}\left(K_{1}+2 K_{2}+2 K_{3}+K_{4}\right), \\
\mathrm{S}_{n+1}(t)=\mathrm{S}_{n}(t)+\frac{h}{6}\left(L_{1}+2 L_{2}+2 L_{3}+L_{4}\right), \\
\mathrm{R}_{n+1}(t)=\mathrm{R}_{n}(t)+\frac{h}{6}\left(M_{1}+2 M_{2}+2 M_{3}+M_{4}\right) .
\end{gathered}
$$

Here,

$$
\begin{aligned}
K_{1}= & -\lambda\langle k\rangle \mathrm{I}_{n}(t) \mathrm{S}_{n}(t)-\beta\langle k\rangle \mathrm{S}_{n}(t) \mathrm{I}_{n}(t) \\
& -\gamma\langle k\rangle \mathrm{S}_{n}(t) \mathrm{I}_{n}(t), \\
K_{2}= & -\lambda\langle k\rangle\left(\mathrm{I}_{n}(t)+\frac{h}{2} K_{1}\right)\left(\mathrm{S}_{n}(t)+\frac{h}{2} L_{1}\right) \\
& -\beta\langle k\rangle\left(\mathrm{S}_{n}(t)+\frac{h}{2} L_{1}\right)\left(\mathrm{I}_{n}(t)+\frac{h}{2} K_{1}\right) \\
& -\gamma\langle k\rangle\left(\mathrm{S}_{n}(t)+\frac{h}{2} L_{1}\right)\left(\mathrm{I}_{n}(t)+\frac{h}{2} K_{1}\right), \\
K_{3}= & -\lambda\langle k\rangle\left(\mathrm{I}_{n}(t)+\frac{h}{2} K_{2}\right)\left(\mathrm{S}_{n}(t)+\frac{h}{2} L_{2}\right) \\
& -\beta\langle k\rangle\left(\mathrm{S}_{n}(t)+\frac{h}{2} L_{2}\right)\left(\mathrm{I}_{n}(t)+\frac{h}{2} K_{2}\right) \\
& -\gamma\langle k\rangle\left(\mathrm{S}_{n}(t)+\frac{h}{2} L_{2}\right)\left(\mathrm{I}_{n}(t)+\frac{h}{2} K_{2}\right),
\end{aligned}
$$

$$
\begin{aligned}
& K_{4}=-\lambda\langle k\rangle\left(\mathrm{I}_{n}(t)+h K_{3}\right)\left(\mathrm{S}_{n}(t)+h L_{3}\right) \\
& -\beta\langle k\rangle\left(\mathrm{S}_{n}(t)+h L_{3}\right)\left(\mathrm{I}_{n}(t)+h K_{3}\right) \\
& -\gamma\langle k\rangle\left(\mathrm{S}_{n}(t)+h L_{3}\right)\left(\mathrm{I}_{n}(t)+h K_{3}\right), \\
& L_{1}=\lambda\langle k\rangle \mathrm{I}_{n}(t) \mathrm{S}_{n}(t)-\alpha\langle k\rangle \mathrm{S}_{n}(t)\left[\mathrm{S}_{n}(t)+\mathrm{R}_{n}(t)\right] \\
& -\eta \mathrm{S}_{n}(t)-\delta \mathrm{S}_{n}(t) \\
& L_{2}=\lambda\langle k\rangle\left(\mathrm{I}_{n}(t)+\frac{h}{2} K_{1}\right)\left(\mathrm{S}_{n}(t)+\frac{h}{2} L_{1}\right) \\
& -\alpha\langle k\rangle\left(\mathrm{S}_{n}(t)+\frac{h}{2} L_{1}\right) \\
& \times\left[\left(\mathrm{S}_{n}(t)+\frac{h}{2} L_{1}\right)+\left(\mathrm{R}_{n}(t)+\frac{h}{2} M_{1}\right)\right], \\
& L_{3}=\lambda\langle k\rangle\left(\mathrm{I}_{n}(t)+\frac{h}{2} K_{2}\right)\left(\mathrm{S}_{n}(t)+\frac{h}{2} L_{2}\right) \\
& -\alpha\langle k\rangle\left(\mathrm{S}_{n}(t)+\frac{h}{2} L_{2}\right) \\
& \times\left[\left(\mathrm{S}_{n}(t)+\frac{h}{2} L_{2}\right)+\left(\mathrm{R}_{n}(t)+\frac{h}{2} M_{2}\right)\right] \\
& -\eta\left(\mathrm{S}_{n}(t)+\frac{h}{2} L_{2}\right)-\delta\left(\mathrm{S}_{n}(t)+\frac{h}{2} L_{2}\right), \\
& L_{4}=\lambda\langle k\rangle\left(\mathrm{I}_{n}(t)+h K_{3}\right)\left(\mathrm{S}_{n}(t)+h L_{3}\right) \\
& -\alpha\langle k\rangle\left(\mathrm{S}_{n}(t)+h L_{3}\right) \\
& \times\left[\left(\mathrm{S}_{n}(t)+h L_{3}\right)+\left(\mathrm{R}_{n}(t)+h M_{3}\right)\right] \\
& -\eta\left(\mathrm{S}_{n}(t)+h L_{3}\right)-\delta\left(\mathrm{S}_{n}(t)+h L_{3}\right), \\
& M_{1}=\alpha\langle k\rangle \mathrm{S}_{n}(t)\left[\mathrm{S}_{n}(t)+\mathrm{R}_{n}(t)\right] \\
& +\delta \mathrm{S}_{n}(t)+\beta\langle k\rangle \mathrm{S}_{n}(t) \mathrm{I}_{n}(t) \\
& +\eta \mathrm{S}_{n}(t)+\gamma\langle k\rangle \mathrm{S}_{n}(t) \mathrm{I}_{n}(t), \\
& M_{2}=\alpha\langle k\rangle\left(\mathrm{S}_{n}(t)+\frac{h}{2} L_{1}\right) \\
& \times\left[\left(\mathrm{S}_{n}(t)+\frac{h}{2} L_{1}\right)+\left(\mathrm{R}_{n}(t)+\frac{h}{2} M_{1}\right)\right] \\
& +\delta\left(\mathrm{S}_{n}(t)+\frac{h}{2} L_{1}\right) \\
& +\beta\langle k\rangle\left(\mathrm{S}_{n}(t)+\frac{h}{2} L_{1}\right)\left(\mathrm{I}_{n}(t)+\frac{h}{2} K_{1}\right) \\
& +\eta\left(\mathrm{S}_{n}(t)+\frac{h}{2} L_{1}\right) \\
& +\gamma\langle k\rangle\left(\mathrm{S}_{n}(t)+\frac{h}{2} L_{1}\right)\left(\mathrm{I}_{n}(t)+\frac{h}{2} K_{1}\right),
\end{aligned}
$$




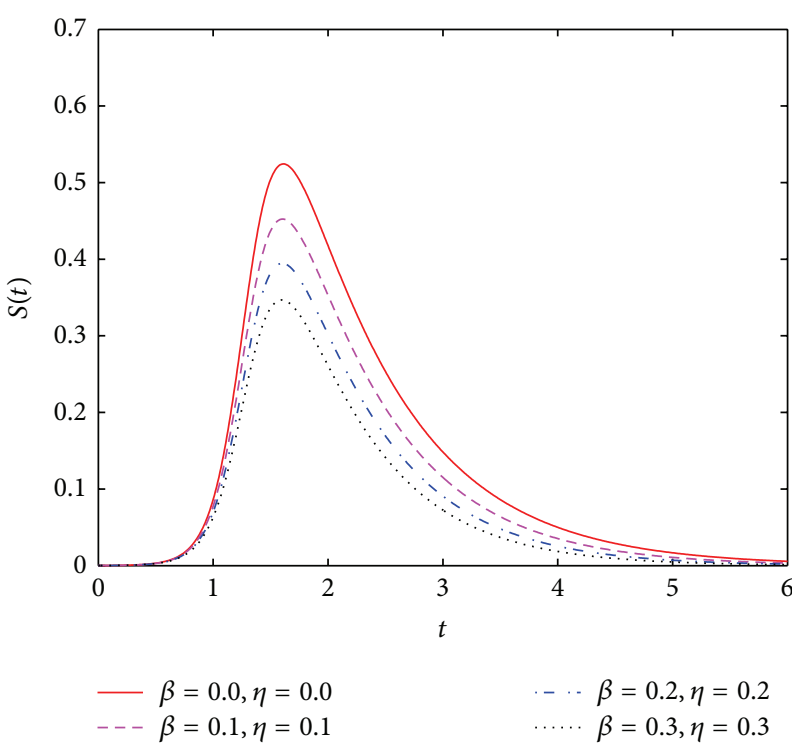

(a) Density of spreaders

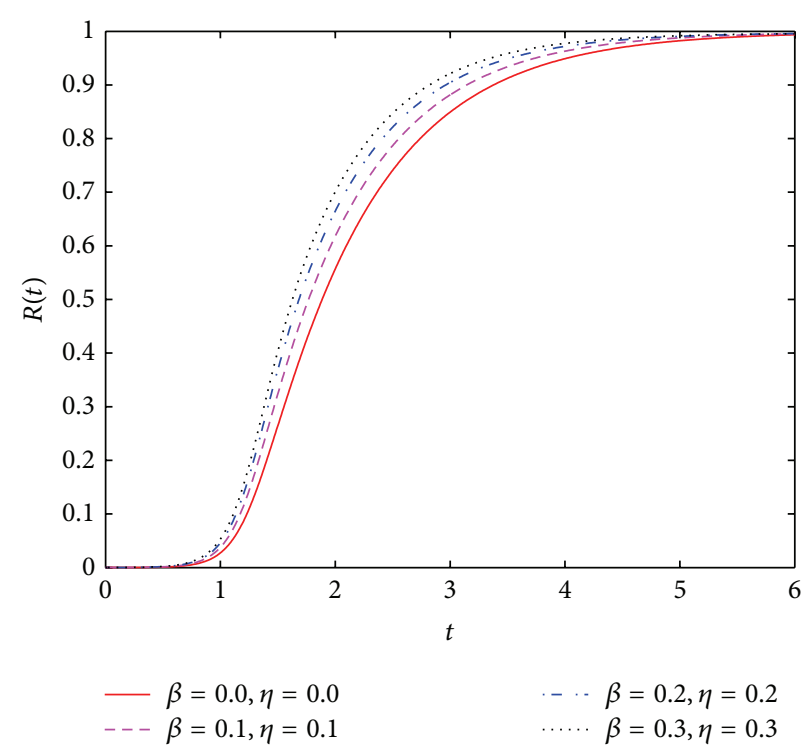

(b) Density of stiflers

Figure 4: (a) The density of spreaders, $\mathrm{S}(t)$, and (b) the influence of the rumor, $\mathrm{R}(t)$, as a function of time for different $\beta$ and $\eta$ with $\lambda=0.7$, $\delta=0.1, \alpha=0.1$, and $\gamma=0.2$, respectively.

$$
\begin{aligned}
M_{3}= & \alpha\langle k\rangle\left(\mathrm{S}_{n}(t)+\frac{h}{2} L_{2}\right) \\
& \times\left[\left(\mathrm{S}_{n}(t)+\frac{h}{2} L_{2}\right)+\left(\mathrm{R}_{n}(t)+\frac{h}{2} M_{2}\right)\right] \\
& +\delta\left(\mathrm{S}_{n}(t)+\frac{h}{2} L_{2}\right) \\
& +\beta\langle k\rangle\left(\mathrm{S}_{n}(t)+\frac{h}{2} L_{2}\right)\left(\mathrm{I}_{n}(t)+\frac{h}{2} K_{2}\right) \\
& +\eta\left(\mathrm{S}_{n}(t)+\frac{h}{2} L_{2}\right) \\
& +\gamma\langle k\rangle\left(\mathrm{S}_{n}(t)+\frac{h}{2} L_{2}\right)\left(\mathrm{I}_{n}(t)+\frac{h}{2} K_{2}\right), \\
M_{4}= & \alpha\langle k\rangle\left(\mathrm{S}_{n}(t)+h L_{3}\right) \\
& \times\left[\left(\mathrm{S}_{n}(t)+h L_{3}\right)+\left(\mathrm{R}_{n}(t)+h M_{3}\right)\right] \\
& +\delta\left(\mathrm{S}_{n}(t)+h L_{3}\right) \\
& +\beta\langle k\rangle\left(\mathrm{S}_{n}(t)+h L_{3}\right)\left(\mathrm{I}_{n}(t)+h K_{3}\right) \\
& +\eta\left(\mathrm{S}_{n}(t)+h L_{3}\right) \\
+ & \gamma\langle k\rangle\left(\mathrm{S}_{n}(t)+h L_{3}\right)\left(\mathrm{I}_{n}(t)+h K_{3}\right), 1,2, h=0.01 . \\
& \\
& \\
&
\end{aligned}
$$

Calculated by the Runge-Kutta method, Figure 4 vividly shows how the density of spreaders and stiflers changes over time steps on homogeneous network before and after the refutation mechanism is considered, respectively. The peak value of spreaders density $\max \{\mathrm{S}(t)\}$ denotes the biggest fraction of spreaders in the process of rumor spreading, which can be used to measure the maximum influence of the rumor. In Figure 4, the red solid line represents the scenario that $\beta=0$ and $\eta=0$; that is, the refuting mechanism is not considered. From Figure 4(a), we can obtain, as the $\beta$ and $\eta$ increase, that the $\max \{\mathrm{S}(t)\}$ decreases; that is, the bigger the refutation rate $(\beta, \eta)$, the smaller the maximum of rumor influence. Figure 4(b) clearly shows that at first the bigger the refutation rate, the bigger the stifler density. But as the time step goes on, the influence of the refutation mechanism on the stifler density becomes remarkably weakened, and the stifler density reaches the same level in the end with different value of refutation rate $(\beta, \eta)$.

Figure 5 shows how the densities of spreaders change over time for different refuting rates. It can be seen from Figure 5(a) that the $\max \{\mathrm{S}(t)\}$ decreases as $\beta$ increases. Figure 5(a) shows that $\beta$ does not affect the point where the number of spreaders increases sharply and only influences the maximum density of spreaders. That is, $\beta$ does not affect the critical rumor threshold but does affect how the density of spreaders changes. From Figure 5(b), we can also obtain that the $\max \{S(t)\}$ decreases as $\eta$ increases. Figure 5(b) shows that $\eta$ is a key determinant of the point where $S(t)$ begins to increase sharply. The larger the value of $\eta$ is, the later this sharp increase occurs. Therefore, if the authorities can take appropriate measures to refute a rumor, this can provide enough time for them to plan how to cope with the impact of the spreading rumor. If we take the buying frenzy for iodized salt that occurred in China as an example, effective refutation of the rumor would have provided time to ensure an adequate supply of iodized salt. This would maintain public calm until the rumor propagation ceases. From Figure 5(a), we can also 

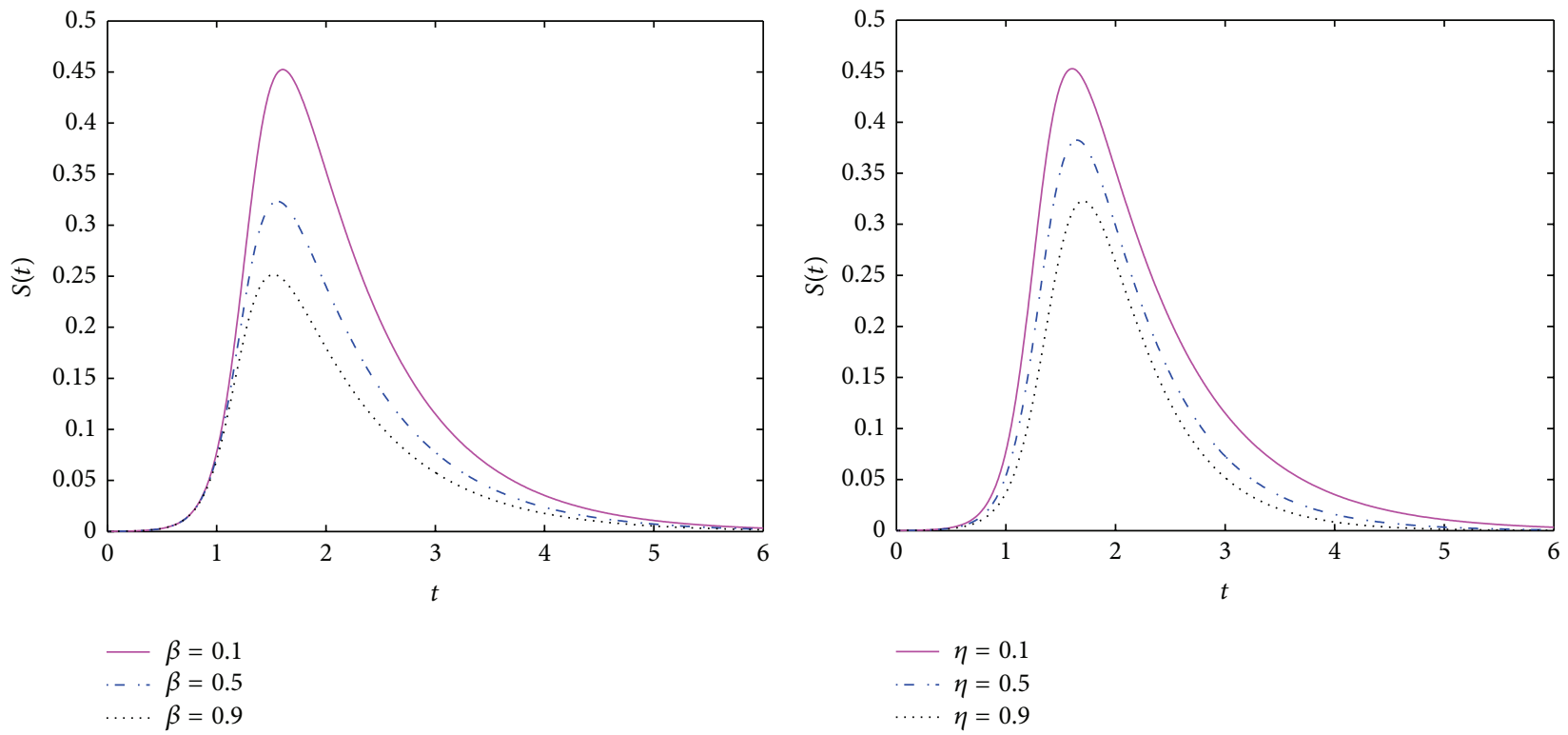

(a) Density of spreaders

(b) Density of spreaders

Figure 5: (a) Density of spreaders over time for different $\beta$ with $\lambda=0.7, \delta=0.1, \alpha=0.1, \eta=0.1$, and $\gamma=0.2$. (b) Density of spreaders over time for different $\eta$ with $\lambda=0.7, \delta=0.1, \alpha=0.1, \beta=0.1$, and $\gamma=0.2$.

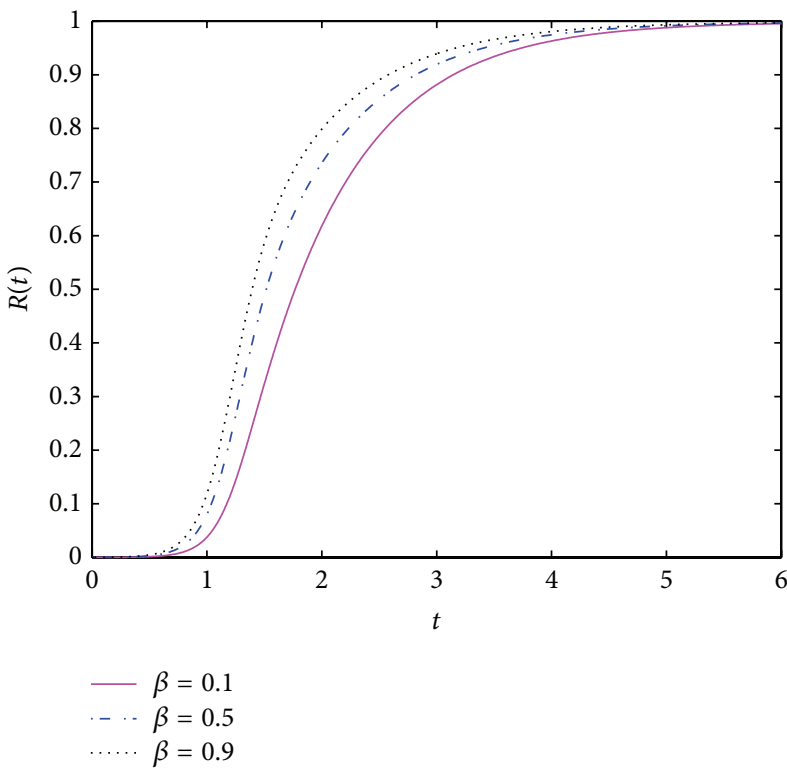

(a) Density of stiflers

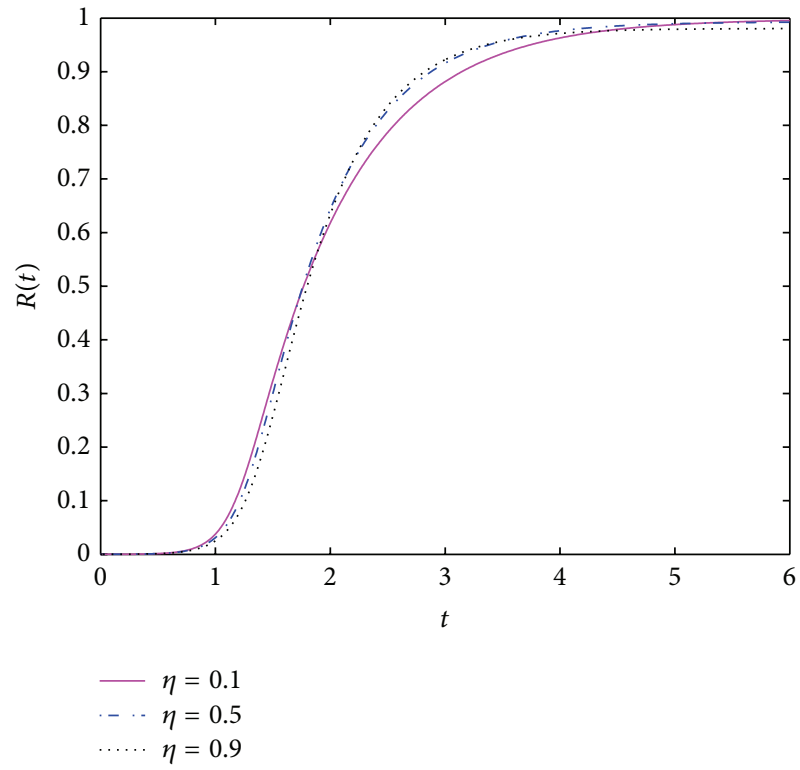

(b) Density of stiflers

Figure 6: (a) Density of stiflers over time for different $\beta$ with $\lambda=0.7, \delta=0.1, \alpha=0.1, \eta=0.1$, and $\gamma=0.2$. (b) Density of stiflers over time for different $\eta$ with $\lambda=0.7, \delta=0.1, \alpha=0.1, \beta=0.1$, and $\gamma=0.2$.

obtain that when $\beta$ value varies from 0.1 to 0.5 , the peak value of spreaders density decreases to 0.14 . However, from Figure 5(b), we can get that when $\eta$ value varies from 0.1 to 0.5 , the peak value of spreaders density only decreases to 0.08 . So from Figure 5, we can obtain that, under the same conditions, increasing the ignorant's refutation rate $\beta$ can reduce the peak value of spreaders density, which is better than increasing the spreader's refutation rate $\eta$.
Figure 6 shows how the densities of stiflers change over time for different refuting rates. Figure $6(a)$ clearly shows that the final density of stiflers is unchangeable as $\beta$ increases. In the process of rumor propagation the bigger the ignorant's refutation rate, the bigger the stifler density. But as the time step goes on, the influence of the ignorant's refutation rate $\beta$ on the stifler density becomes remarkably weakened, and the stifler density reaches the same level in the end with different 
value of the ignorant's refutation rate $\beta$. From Figure 6(b), we can obtain that the final density of stiflers decreases as $\eta$ increases. That is, the larger the value of $\eta$, the smaller the final influence of rumor propagation.

The results of these simulations demonstrate that the refutation strategies adopted by the authorities play an important role in rumor-propagation process. When rumor prevails, the authorities can improve the refuting rate by a variety of ways for reducing the influence of rumors propagation. For example, the authorities should strengthen construction of refuting rumor websites to improve the rumor identification ability of the public; the authorities should choose a wide variety of mass media to release the rumor truth to the public. Particularly, in the days of information and network, apart from the older media (the newspaper, broadcast, TV, and so on), the authorities attach special importance on the application of new media such as Twitter and Forum. The microblogging of the authorities can be used to timely refute the rumor and quickly provide a smooth flow of correct information to the public, thereby helping them to learn the truth, which can improve the refuting rate. At the same time, the authorities should strengthen the education investment to improve the rumor identification of the public, which also can increase the refuting rate in the rumor model. The authorities can strengthen surveillance and monitoring of the public opinion to early discover the rumor and then can timely refute the rumor to reduce the influence of rumor. For more important is that the authorities should enhance the government credibility. The public believe more the truth of rumor which is released by the authorities of high credibility. The above methods would bring about a high refutation rate that can reduce the influence of rumor and mitigate the damage it can cause.

\section{Conclusions}

In this paper, we analyzed the dynamics of rumor propagation in homogeneous networks using a model that incorporated a refutation mechanism. We found the following novel features and significant results of the model.

(1) To account for differences in the effectiveness of the strategies adopted by the authorities, thereby making the simulations more realistic, we incorporated a refutation mechanism in the rumor-propagation model. We then derived mean-field equations to describe changes in the proportions of spreaders, ignorants, and stiflers. The classical SIR rumor-spread model and Nekovee's rumor-spread model with a forgetting mechanism are special cases of the model.

(2) Our steady-state analysis revealed the existence of critical threshold for rumor propagation. In particular, for $\delta=1$, it is only dependent on the average degree of the network $\langle k\rangle$ and the refutation rate $\eta$. Our model allows prediction of $\mathrm{R}$ for a rumor with different propagation rates $\lambda$ and $\eta$. Graphs of the results confirmed the existence of the threshold and that it is dependent on both $\langle k\rangle$ and $\eta$.
(3) We used the Runge-Kutta method to simulate the mean-field equations used in the rumor model with a refutation mechanism. The simulations showed that, under a given set of conditions, the refutation factor affected the rumor-propagation process: an increasing refutation rate decreased the maximum spreader density.

Our results suggest that the authorities should choose appropriate media to increase the refutation factor by releasing the truth to the public. Using mass media with a high audience rating among the individuals in the population who are most likely to spread and receive the rumor and using a medium with high credibility for these individuals would generate a high refutation rate that can effectively slow the rumor's propagation and mitigate the damage it can cause.

The present study only addressed some of these aspects. In complex networks, the incorporation of refutation mechanisms in models of rumor propagation will be an interesting topic for future research.

\section{Conflict of Interests}

The authors declare that there is no conflict of interests regarding the publication of this paper.

\section{Acknowledgments}

This study is supported by Grants from the National Natural Science Foundation of China (project no. 90924030) and Social Development of Metropolis and Construction of Smart City (project no. 085SHDX001).

\section{References}

[1] S. Galam, "Modelling rumors: the no plane Pentagon French hoax case," Physica A: Statistical Mechanics and Its Applications, vol. 320, pp. 571-580, 2003.

[2] M. Kosfeld, "Rumours and markets," Journal of Mathematical Economics, vol. 41, no. 6, pp. 646-664, 2005.

[3] H. Hayakawa, "Ryugen no shakaigaku—keishikisyakaigaku karano sekkin (sociology of rumor approach from formal sociology)," Seikyusya, Tokyo, Japan, in Japanese, 2002.

[4] S. A. Thomas, "Lies, damn lies, and rumors: an analysis of collective efficacy, rumors, and fear in the wake of Katrina," Sociological Spectrum, vol. 27, no. 6, pp. 679-703, 2007.

[5] E. R. Danzig, P. W. Thayer, and L. R. Galanter, The Effects of a Threatening Rumor on a Disaster-Stricken Community, National Academy of Science, National Research Council, Washington, DC, USA, 1958.

[6] Z. L. Zhang and Z. Q. Zhang, "An interplay model for rumour spreading and emergency development," Physica A: Statistical Mechanics and its Applications, vol. 388, no. 19, pp. 4159-4166, 2009.

[7] D. J. Daley and D. G. Kendall, "Epidemics and rumours," Nature, vol. 204, no. 4963, p. 1118, 1964.

[8] D. P. Maki and M. Thompson, Mathematical Models and Applications, Prentice-Hall, Englewood Cliffs, NJ, USA, 1973. 
[9] D. P. Maki, Mathematical Models and Applications, with Emphasis on Social, Life, and Management Sciences, Prentice Hall, Englewood Cliffs, NJ, USA, 1973.

[10] J. D. Murray, Mathematical Modeling in Epidemiology, Springer, Berlin, Germany, 1980.

[11] A. Sudbury, "The proportion of the population never hearing a rumour," Journal of Applied Probability, vol. 22, no. 2, pp. 443446, 1985.

[12] B. Doerr, M. Fouz, and T. Friedrich, "Social networks spread rumors in sublogarithmic time," in Proceedings of the 43rd ACM Symposium on Theory of Computing, pp. 21-30, 2011.

[13] D. Trpevski, W. K. S. Tang, and L. Kocarev, "Model for rumor spreading over networks," Physical Review E, vol. 81, no. 5, Article ID 056102, 2010.

[14] F. Roshani and Y. Naimi, "Effects of degree-biased transmission rate and nonlinear infectivity on rumor spreading in complex social networks," Physical Review E, Statistical, Nonlinear, and Soft Matter Physics, vol. 85, no. 3, Article ID 036109, 2012.

[15] J. Huang and X. Jin, "Preventing rumor spreading on smallworld networks," Journal of Systems Science and Complexity, vol. 24, no. 3, pp. 449-456, 2011.

[16] P. Li and Q. Z. Zhao, "Rumor spreading in local-world evolving network," Communications in Computer and Information Science, vol. 227, pp. 693-699, 2011.

[17] D. H. Zanette, "Critical behavior of propagation on small-world networks," Physical Review E-Statistical, Nonlinear, and Soft Matter Physics, vol. 64, no. 5, Article ID 050901, 4 pages, 2001.

[18] D. H. Zanette, "Dynamics of rumor propagation on small-world networks," Physical Review E, vol. 65, no. 4, Article ID 041908, pp. 1-9, 2002.

[19] L. Buzna, K. Peters, and D. Helbing, "Modelling the dynamics of disaster spreading in networks," Physica A: Statistical Mechanics and its Applications, vol. 363, no. 1, pp. 132-140, 2006.

[20] Y. Moreno, M. Nekovee, and A. F. Pacheco, "Dynamics of rumor spreading in complex networks," Physical Review E, Statistical, Nonlinear, and Soft Matter Physics, vol. 69, no. 6, Article ID 066130, pp. 1-66130, 2004.

[21] J. Gu and X. Cai, "The forget-remember mechanism for 2-state spreading," http://arxiv.org/pdf/nlin/0702021.pdf.

[22] J. Gu, W. Li, and X. Cai, "The effect of the forget-remember mechanism on spreading," European Physical Journal B, vol. 62, no. 2, pp. 247-255, 2008.

[23] M. Nekovee, Y. Moreno, G. Bianconi, and M. Marsili, "Theory of rumour spreading in complex social networks," Physica A, vol. 374, no. 1, pp. 457-470, 2007.

[24] L. Zhao, Q. Wang, J. Cheng, Y. Chen, J. Wang, and W. Huang, "Rumor spreading model with consideration of forgetting mechanism: a case of online blogging LiveJournal," Physica A: Statistical Mechanics and Its Applications, vol. 390, no. 13, pp. 2619-2625, 2011.

[25] L. J. Zhao, J. J. Wang, Y. C. Chen, Q. Wang, J. Cheng, and H. Cui, "SIHR rumor spreading model in social networks," Physica A: Statistical Mechanics and Its Applications, vol. 391, no. 7, pp. 2444-2453, 2012.

[26] L. Zhao, X. Qiu, X. Wang, and J. Wang, "Rumor spreading model considering forgetting and remembering mechanisms in inhomogeneous networks," Physica A: Statistical Mechanics and Its Applications, vol. 392, no. 4, pp. 987-994, 2013.

[27] L. J. Zhao, X. L. Wang, X. Y. Qiu, and J. J. Wang, "A model for the spread of rumors in BARrat-BARthelemy-Vespignani (BBV) networks," Physica A: Statistical Mechanics and its Applications, vol. 392, no. 21, pp. 5542-5551, 2013.
[28] F. Xiong, Y. Liu, Z. Zhang, J. Zhu, and Y. Zhang, "An information diffusion model based on retweeting mechanism for online social media," Physics Letters A: General, Atomic and Solid State Physics, vol. 376, no. 30-31, pp. 2103-2108, 2012.

[29] J. Cheng, Y. Liu, B. Shen, and W. Yuan, "An epidemic model of rumor diffusion in online social networks," The European Physical Journal B. Condensed Matter and Complex Systems, vol. 86, no. 1, article 29, 7 pages, 2013. 


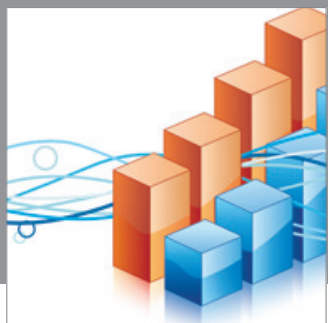

Advances in

Operations Research

mansans

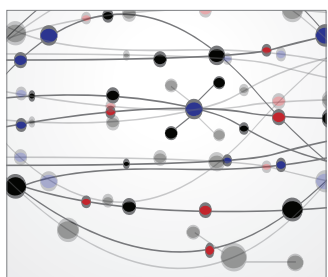

The Scientific World Journal
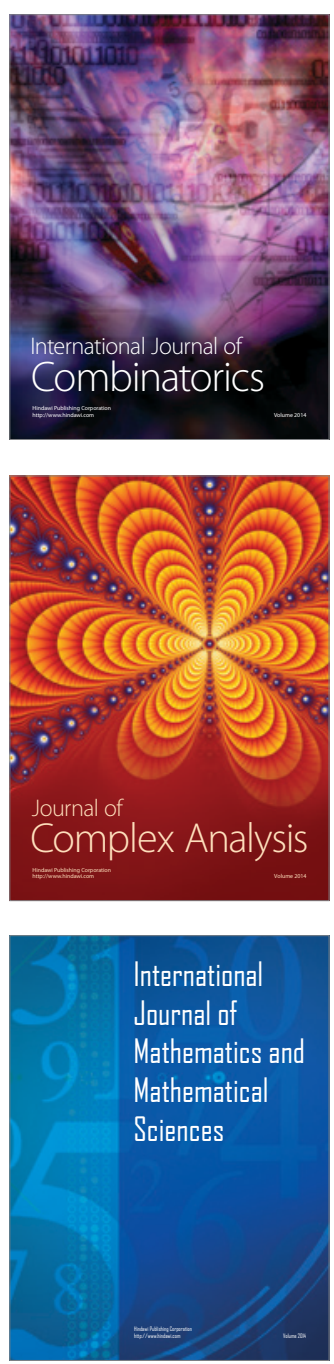
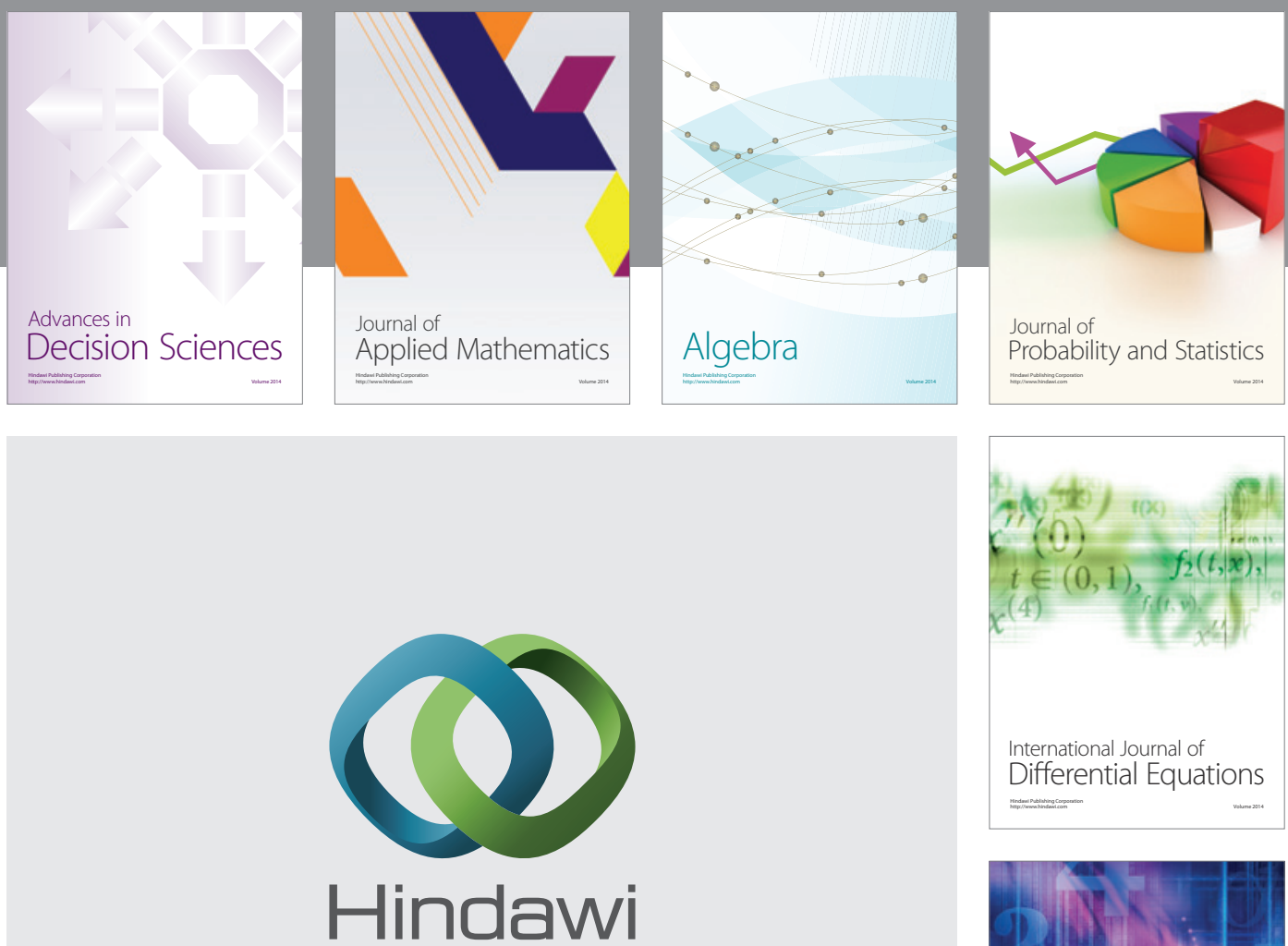

Submit your manuscripts at http://www.hindawi.com
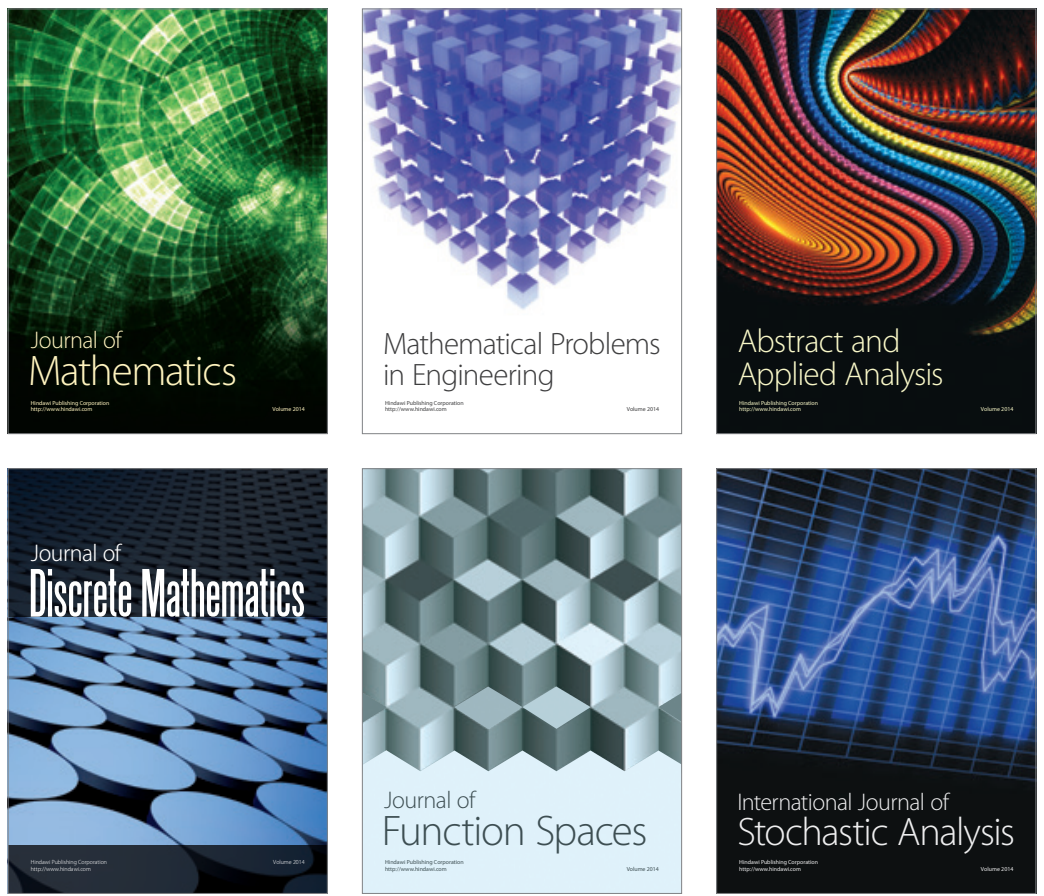

Journal of

Function Spaces

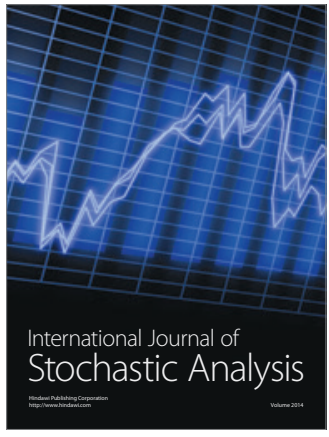

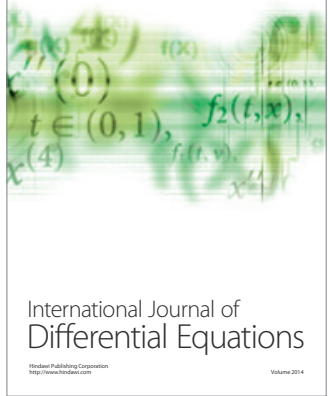
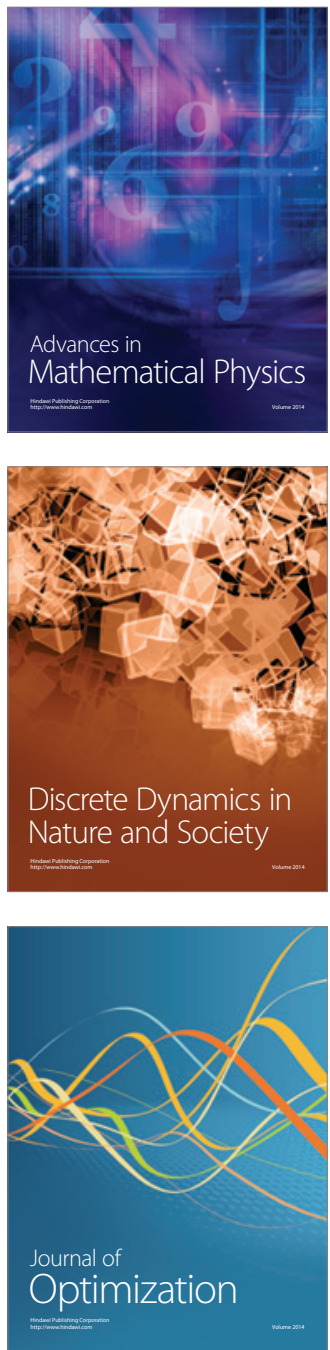